\title{
Research Paper \\ Identifying Qualitative Factors Affecting the Quality of Clinical Education
}

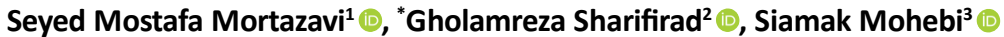

1. Department of Educational Management, Faculty of Humanities, Qom Branch, Islamic Azad University, Qom, Iran.

2. Department of Social Sciences, Faculty of Humanities, Islamic Azad University, Qom Branch, Islamic Azad University, Qom, Iran.

3. Department of Health Sciences, School of Health, Qom University of Medical Sciences, Qom, Iran.

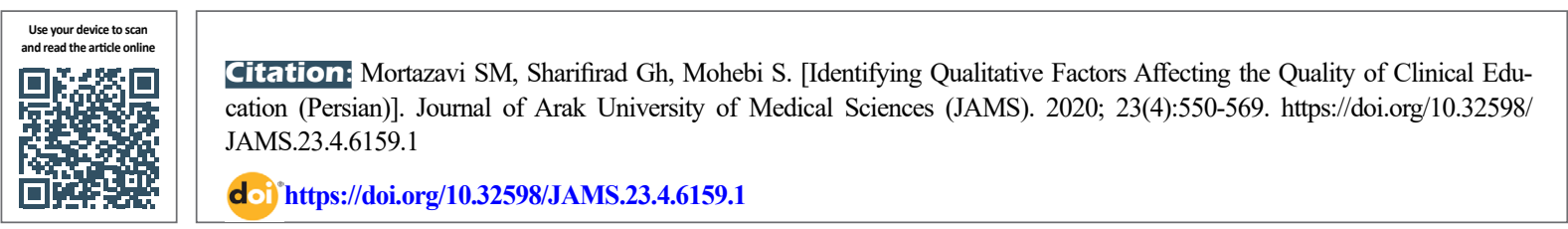

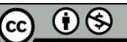

Article Info:

Received: 24 Apr 2020

Accepted: 11 Jul 2020

Available Online: 01 Oct 2020

Keywords:

Clinical education, Quality of education, Hospitals in Saveh City

\section{A B STRACT}

Background and Aim Training clinical students who learn the theoretical lessons in hospitals in practice will play an important role in improving treatment processes and providing better services to patients. Therefore, the purpose of this study is to identify the factors affecting the quality of clinical education in hospitals in Saveh City.

Methods \& Materials This research is a combination of exploratory studies and exploratory projects. In the qualitative stage, the qualitative method of Grand Strauss and Corbyn 1998 was used to explain the quality of clinical training. Since the students who have taken clinical courses in the Faculty of Medical Sciences of Saveh Hospitals in 1398-1397 are about 200 people and the professors and educators who teach these people are about 20 people, which according to Karajsi and Morgan, the sample size should be 140, of which 20 were interviewed and 120 were filled out with paper and electronic questionnaires. In this study, semi-structured interviews, and a researcher-made questionnaire with Cronbach's alpha coefficient of 0.598 were selected as the data collection method. This research was performed using the qualitative method and using MAZQDA 10 and SPSS 26 software, the items obtained from qualitative research were collected and exploratory factor analysis was used to identify the factors.

Ethical Considerations Considering that this study is one of the research priorities of Saveh School of Medical Sciences, it has a research license letter (No. 24.127. D.99).

Results The results showed that the factors affecting the quality of clinical education include five factors. The first factor is space and facilities, the second factor is the improvement of educational processes, the third factor is educational and managerial deficiencies, the fourth factor is the evaluation of the teacher and the fifth factor is the qualitative improvement of education.

Conclusion According to the quantitative and qualitative results of this study and by interviewing twenty experienced professors and student representatives, the factors affecting the quality of clinical education in Saveh hospitals were identified.

\section{Extended Abstract}

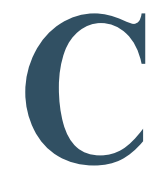

\section{Introduction}

linical education is one of the most important pillars of medical students' edu- cation. Because this education is the operational stage of theoretical education, it should be able to institutionalize the learned knowledge in learners with the help of experienced professors and hospital facilities in a way that has the most impact. Clinical education is the most important part of medical education, which is an integral part of it, which

\section{Corresponding Author:}

\section{Gholamreza Sharifirad, PhD.}

Address: Department of Social Sciences, Faculty of Humanities, Islamic Azad University, Qom Branch, Qom, Iran.

Tel: +98 (912) 2563284

E-mail: dr.sharifirad@gmail.com 
is known as the heart of vocational education. During clinical training, what is learned is put into practice, skills are taught, and students have confronted with the realities of the workplace and this study aim to explore the qualitative factors in clinical education provided in Saveh hospitals because each region has specific cultures and educational processes, as well as problems in each university, are different that justifies the need for this research.

\section{Materials and Methods}

To understand the phenomenon, this research starts with qualitative data, and then the secondary or quantitative stage is made. Therefore, this study is a combination of studies and exploratory projects. In the qualitative stage, to explain the quality of clinical education, the qualitative method of Grand Strauss and Corbin 1998 was used. Considering that the students who took clinical courses in the Faculty of Medical Sciences in 2018-2019 were about 200 people and the professors and instructors who teach these people were about 20 people, according to Karajsi and Morgan table the sample size should be 140 people, of which 20 participated in the interview and 120 people filled out a paper and electronic questionnaire. In this study, semistructured interviews were selected as the method of data collection. Each interview was then coded. The interviews were completely and accurately transcribed using MAXQDA 10 software while collecting information, and the initial coding was done at the same time in the initial coding, the researcher examined the transcribed or typed data line by line as units of analysis, and the coding was performed based on qualitative findings.

\section{Results}

Considering that the $\mathrm{KMO}$ value was equal to 0.860 and the significance level of the Bartlett sphericity test was less than 0.01 , the reliability was also calculated that the total reliability of the questionnaire in this study was 0.958 , which is excellent. And the results of this research are as follows:
First Factor: space, facilities, shortages, and educational needs that the nutrition factor and welfare facilities with the number 0.774 and training rooms in the wards of hospitals with 0.761 , and the use of equipment and consumables of hospitals 0.729 . These were among the three main factors that had the highest scores.

Second Factor: Results and consequences of improving educational processes that increase the level of patient safety with a score of 0.806 and increase patient satisfaction with a score of 0.776 , and achieve hospital goals and customer satisfaction with a score of 0.747 .

Third Factor: educational and managerial deficiencies, lack of a specific lesson plan with a score of 0.762 , lack of a stable faculty member with a score of 0.743 , and the factor of students' participation with professors in educational processes with a score of 0.741 .

Fourth Factor: The correct cases of educational evaluation that the experienced teacher with a score of 0.806 , the teacher and students with a participatory spirit with a score of 0.756 , and the factor that clinical teachers have a suitable quality with a score of 0.741 .

Fifth Factor: Improving the quality of education and improving educational processes, which is the factor of using material and spiritual motivations for students with a score of 0.840 , the factor of using new educational facilities in clinical education with a score of 0.825 , and using in-service training classes to empower teachers with a score of 0.802 obtained the highest scores (Tables 1 \& 2).

\section{Discussion and Conclusion}

This study aims to identify the factors affecting the quality of clinical education in hospitals in Saveh City. According to the quantitative and qualitative results of this research and by interviewing twenty experienced professors and student representatives, the first factor is space and facilities and shortages and educational needs, which is the factor of nutrition and welfare facilities with a score of 0.774 and training rooms in hospital wards with 0.761 and the use of

Table 1. Results of KMO test and Bartlett sphericity test

\begin{tabular}{|c|c|c|}
\hline \multicolumn{2}{|c|}{ KMO test } & \multirow{2}{*}{$\begin{array}{r}0.860 \\
4980.8\end{array}$} \\
\hline & Chi-square & \\
\hline \multirow[t]{2}{*}{ Bartlett spherical test } & Degrees of freedom & 1540 \\
\hline & Significance level & $<0.01$ \\
\hline
\end{tabular}


Table 1. Basic statistical characteristics of the questionnaire by PC method

\begin{tabular}{|c|c|c|c|}
\hline Factors & Special Value & Percentage of Variance & Compression Percentage \\
\hline 1 & 18.11 & 32.34 & 32.34 \\
\hline 2 & 4.30 & 7.68 & 40.02 \\
\hline 3 & 3.26 & 7.61 & 47.62 \\
\hline 4 & 2.51 & 6.27 & 53.89 \\
\hline 5 & 1.80 & 5 & 58.89 \\
\hline 6 & 0.99 & 2.43 & 61.32 \\
\hline 7 & 0.98 & 2.28 & 63.60 \\
\hline 8 & 0.95 & 2.1 & 65.70 \\
\hline 9 & 0.92 & 1.88 & 67.58 \\
\hline 10 & 0.91 & 1.78 & 69.36 \\
\hline 11 & 0.88 & 1.68 & 71.04 \\
\hline 12 & 0.86 & 1.63 & 72.67 \\
\hline 13 & 0.84 & 1.58 & 74.25 \\
\hline 14 & 0.81 & 1.52 & 75.77 \\
\hline 15 & 0.80 & 1.44 & 77.21 \\
\hline 16 & 0.78 & 1.40 & 78.61 \\
\hline 17 & 0.71 & 1.27 & 79.88 \\
\hline 18 & 0.69 & 1.23 & 81.11 \\
\hline 19 & 0.68 & 1.21 & 82.32 \\
\hline 20 & 0.65 & 1.15 & 83.47 \\
\hline 21 & 0.61 & 1.09 & 84.55 \\
\hline 22 & 0.56 & 1 & 85.56 \\
\hline 23 & 0.51 & 0.91 & 86.47 \\
\hline 24 & 0.48 & 0.86 & 87.33 \\
\hline 25 & 0.47 & 0.83 & 88.16 \\
\hline 26 & 0.46 & 0.82 & 88.98 \\
\hline 27 & 0.44 & 0.79 & 89.77 \\
\hline 28 & 0.40 & 0.72 & 90.48 \\
\hline 29 & 0.37 & 0.66 & 91.14 \\
\hline 30 & 0.35 & 0.63 & 91.77 \\
\hline 31 & 0.34 & 0.62 & 92.39 \\
\hline 32 & 0.32 & 0.58 & 92.97 \\
\hline
\end{tabular}




\begin{tabular}{|c|c|c|c|}
\hline Factors & Special Value & Percentage of Variance & Compression Percentage \\
\hline 33 & 0.31 & 0.55 & 93.52 \\
\hline 34 & 0.30 & 0.54 & 94.06 \\
\hline 35 & 0.29 & 0.51 & 94.57 \\
\hline 36 & 0.27 & 0.48 & 95.06 \\
\hline 37 & 0.24 & 0.43 & 95.48 \\
\hline 38 & 0.22 & 0.40 & 95.88 \\
\hline 39 & 0.21 & 0.39 & 96.27 \\
\hline 40 & 0.20 & 0.36 & 96.63 \\
\hline 41 & 0.19 & 0.35 & 96.98 \\
\hline 42 & 0.18 & 0.33 & 97.31 \\
\hline 43 & 0.17 & 0.31 & 97.62 \\
\hline 44 & 0.16 & 0.28 & 97.90 \\
\hline 45 & 0.15 & 0.26 & 98.16 \\
\hline 46 & 0.14 & 0.26 & 98.42 \\
\hline 47 & 0.12 & 0.22 & 98.64 \\
\hline 48 & 0.12 & 0.22 & 98.86 \\
\hline 49 & 0.12 & 0.22 & 99.08 \\
\hline 50 & 0.10 & 0.19 & 99.26 \\
\hline 51 & 0.09 & 0.16 & 99.42 \\
\hline 52 & 0.08 & 0.14 & 99.56 \\
\hline 53 & 0.07 & 0.13 & 99.69 \\
\hline 54 & 0.07 & 0.13 & 99.81 \\
\hline 55 & 0.06 & 0.1 & 99.91 \\
\hline 56 & 0.05 & 0.09 & 100 \\
\hline
\end{tabular}

equipment and consumables of hospitals 0.729 . These are among the three main factors that have the highest scores.

The second factor is the results and consequences of improving educational processes that increase the level of patient safety with a score of 0.806 and increase patient consent with a score of 0.776 and achieve hospital goals and customer satisfaction with a score of 0.747 .

The third factor is educational and managerial deficiencies with the lack of a specific lesson plan with a score of
0.762 , the lack of a stable faculty with a score of 0.743 and the factor of students' participation with professors in educational processes with a score of 0.741 .

The fourth factor is the teacher evaluation, and the correct cases of educational evaluation in which the experienced teacher with a score of 0.806 , the teacher with the students have a participatory spirit with a score of 0.756 and the factor that the professors of clinical education have a suitable quality have a score of 0.741 . 
Table 2. Factor matrix, rotating factors, set of 56 questions by Varimax method

\begin{tabular}{|c|c|c|c|c|c|}
\hline \multirow{2}{*}{ Questions } & \multicolumn{5}{|c|}{ Factors } \\
\hline & First & Second & Third & Forth & Fifth \\
\hline 20 & 0.774 & & & & \\
\hline 14 & 0.761 & & & & \\
\hline 22 & 0.729 & & & & \\
\hline 12 & 0.717 & & & & \\
\hline 15 & 0.680 & & & & \\
\hline 17 & 0.678 & & & & \\
\hline 13 & 0.674 & & & & \\
\hline 9 & 0.652 & & & & \\
\hline 16 & 0.646 & & & & \\
\hline 11 & 0.640 & & & & \\
\hline 21 & 0.633 & & & & \\
\hline 8 & 0.624 & & & & \\
\hline 18 & 0.610 & & & & \\
\hline 10 & 0.602 & & & & \\
\hline 19 & 0.600 & & & & \\
\hline 25 & 0.584 & & & & \\
\hline 23 & 0.584 & & & & \\
\hline 24 & 0.577 & & & & \\
\hline 51 & & 0.806 & & & \\
\hline 45 & & 0.776 & & & \\
\hline 53 & & 0.747 & & & \\
\hline 56 & & 0.734 & & & \\
\hline 54 & & 0.715 & & & \\
\hline 44 & & 0.701 & & & \\
\hline 52 & & 0.683 & & & \\
\hline 47 & & 0.681 & & & \\
\hline 48 & & 0.667 & & & \\
\hline 55 & & 0.665 & & & \\
\hline 50 & & 0.647 & & & \\
\hline 49 & & 0.599 & & & \\
\hline
\end{tabular}




\begin{tabular}{|c|c|c|c|c|c|}
\hline \multirow{2}{*}{ Questions } & \multicolumn{5}{|c|}{ Factors } \\
\hline & First & Second & Third & Forth & Fifth \\
\hline 46 & & 0.533 & & & \\
\hline 31 & & & 0.762 & & \\
\hline 26 & & & 0.743 & & \\
\hline 32 & & & 0.741 & & \\
\hline 33 & & & 0.732 & & \\
\hline 29 & & & 0.707 & & \\
\hline 36 & & & 0.679 & & \\
\hline 27 & & & 0.664 & & \\
\hline 30 & & & 0.656 & & \\
\hline 28 & & & 0.648 & & \\
\hline 34 & & & 0.637 & & \\
\hline 35 & & & 0.626 & & \\
\hline 37 & & & 0.599 & & \\
\hline 4 & & & & 0.806 & \\
\hline 5 & & & & 0.756 & \\
\hline 1 & & & & 0.739 & \\
\hline 3 & & & & 0.736 & \\
\hline 7 & & & & 0.732 & \\
\hline 6 & & & & 0.724 & \\
\hline 2 & & & & 0.698 & \\
\hline 41 & & & & & 0.840 \\
\hline 42 & & & & & 0.825 \\
\hline 38 & & & & & 0.802 \\
\hline 39 & & & & & 0.799 \\
\hline 40 & & & & & 0.771 \\
\hline 43 & & & & & 0.701 \\
\hline
\end{tabular}

The fifth factor is to improve the quality of education and improve the educational processes, which is the factor of using material and spiritual motivations for students with a score of 0.840 , the factor of using new educational facilities in clinical education with a score of 0.825 and using educational classes. While serving to empower the professors with a score of 0.802 , they obtained the highest scores, and since different factors and even the prevailing cultures in each university are different from each other, it is not possible to prescribe a single version for everyone, 
but research results can be used to synergize knowledge and awareness for other research.

\section{Ethical Considerations}

Compliance with ethical guidelines

This study was approved by the Research Priorities of Saveh School of Medical Sciences, (Code: 24.127. D.99).

Funding

The present paper was extracted from the $\mathrm{PhD}$. dissertation of the first author, Department of Educational Management, Faculty of Humanities, Qom Branch, Islamic Azad University, Qom.

\section{Authors' contributions}

Conceptualization, Methodology: Seyed Mostafa Mortazavi, Gholamreza Sharifirad; Data analysis, Writing original draft, and Writing - review \& editing: All Authors.

\section{Conflicts of interest}

The authors declared no conflict of interest. 


\title{
شناسايى عوامل كيفى مؤثر بر كيفيت آموزش بالينى در بيمارستانهاى شهر ساوه در سالهاى irqu g irav
}

\author{
سيدمصطفى مرتضوى' (1)، "غلامرضا شريفىراد' (1)، سيامك محبى \\ I. كروه مديريث أموزشى، دانشكده علوم انسانى، واحد قمه، دانشكاه آزاد اسلامى، قمه، ايران.

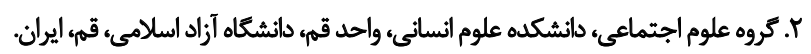

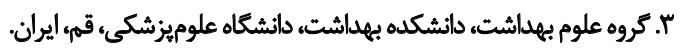

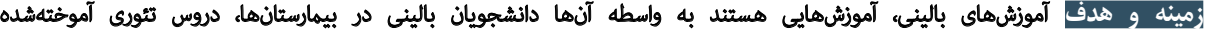

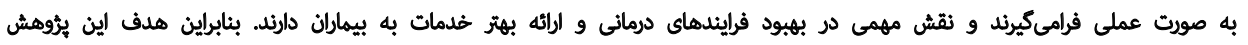

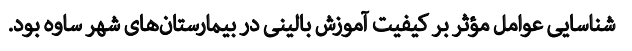

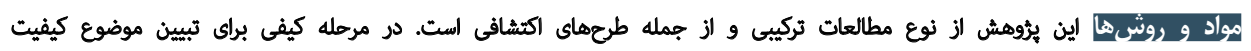

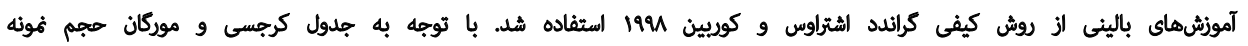
if.

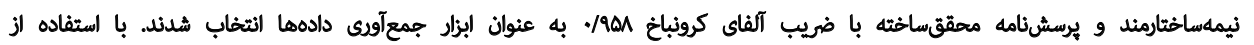

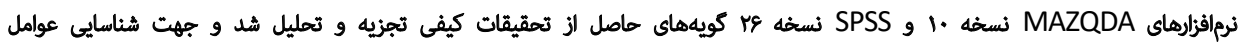
از تحليل عاملى اكتشافى استفاده شد. هلاحظات اخلاقيى اين ئروهش طبق نامه شماره

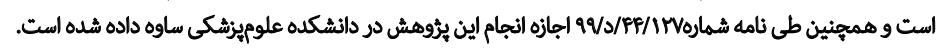

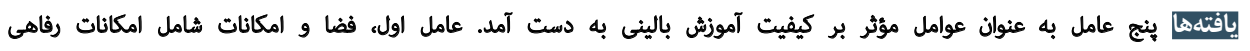

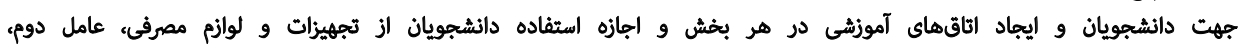

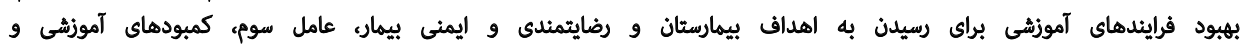

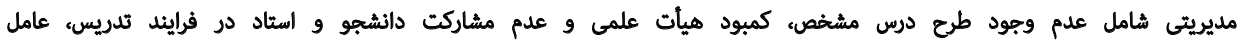

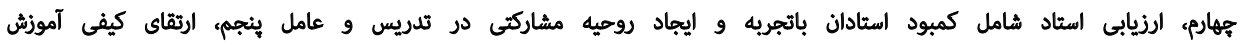

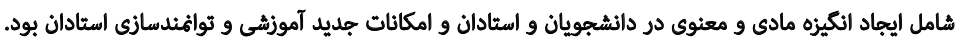

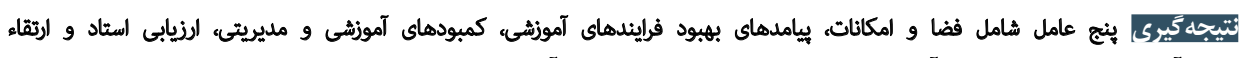

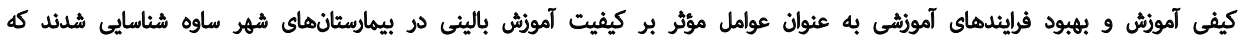

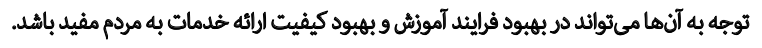

\author{
اطلاعات مقاله:

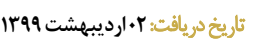

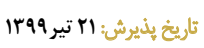

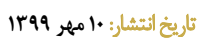

قلب آموزش حرفهايى شناخته شده است. طي آموزش بالينى

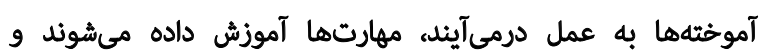

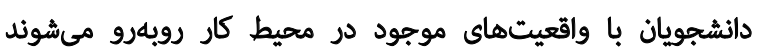

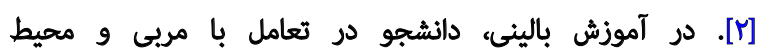

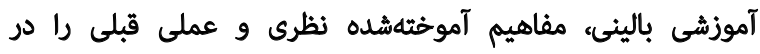

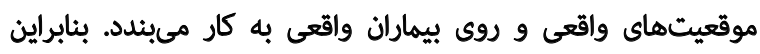

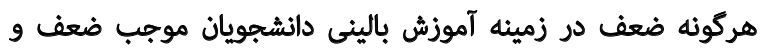

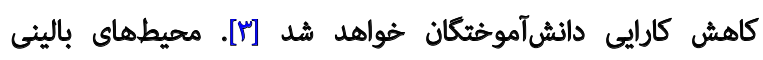

آموزش بالينى يكى از مهمترين اركان آموزش دانشجويان كروه

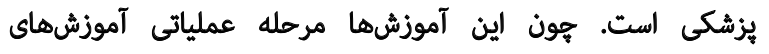

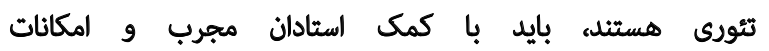

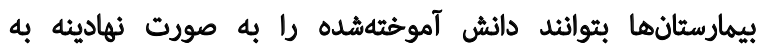

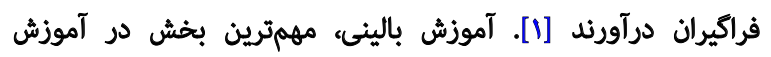

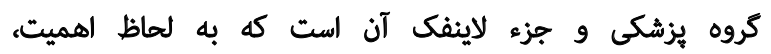

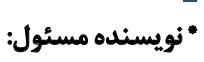

$$
\begin{aligned}
& \text { دكتر غلامرضا شريفى فئداد }
\end{aligned}
$$

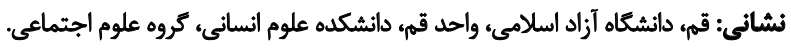

$$
\begin{aligned}
& \text { تلفن: Q } \\
& \text { يست الكترونيكي: dr.sharifirad@gmail.com }
\end{aligned}
$$


در مطالعهاى ديكر، برليز' به بررسى نقش تكنيكهاى جديد

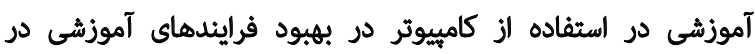

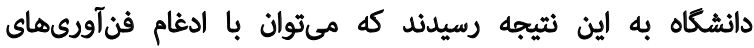

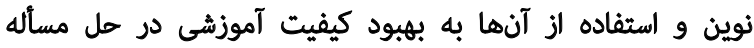

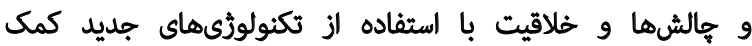

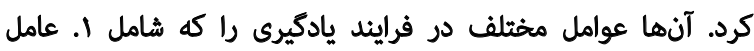

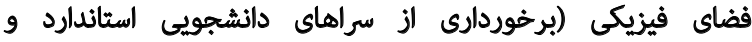

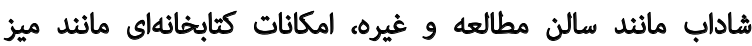

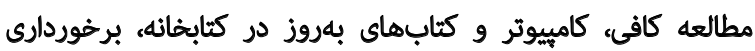

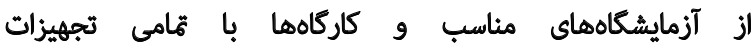
آن، برخوردارى از فضاي سبز و فئائ محيط شاداب دانشكاهي،

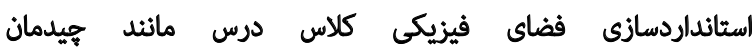

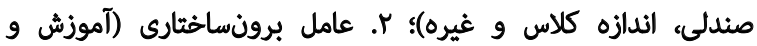

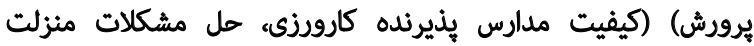

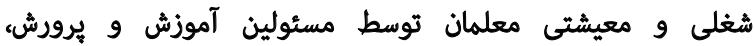

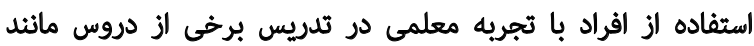

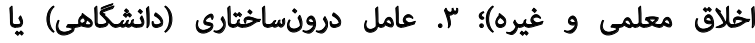

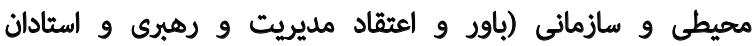

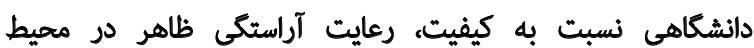

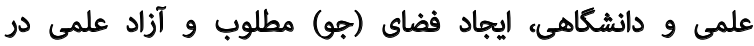

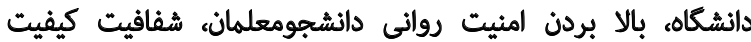

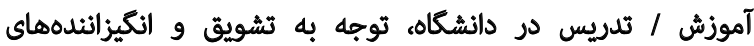

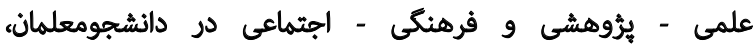

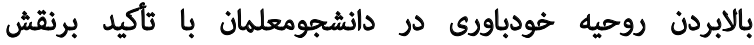

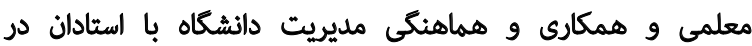

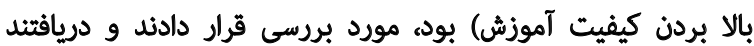

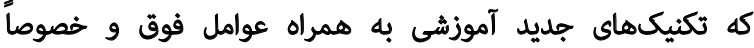

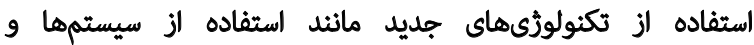

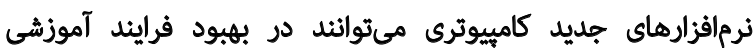

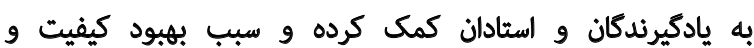

حل جالشها و ايجاد نوآورى و خلاقيت در فراكيران شوند [IT]

سارا هاروى و همكاران در سال 19.19 مطالعهاي تحت عنوان

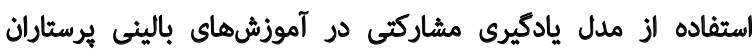

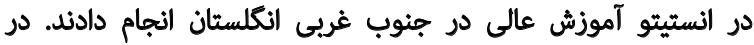

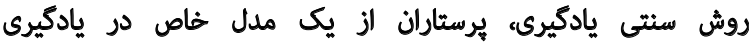

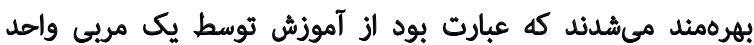

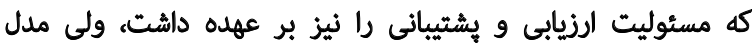

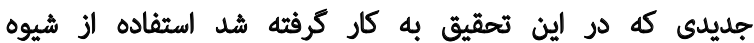

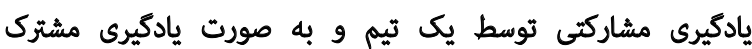

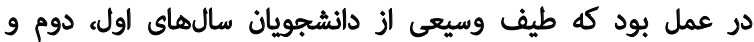

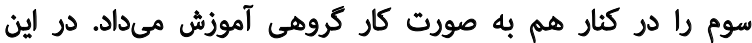

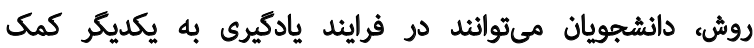

1. Berliner
داراى ويرٔكىهاى متغير و غيرقابل ويشيينى هستند و همين امر

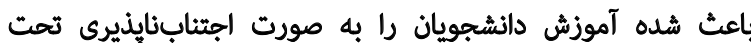

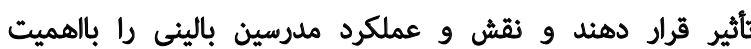

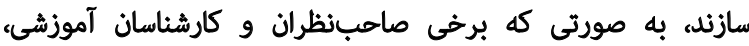

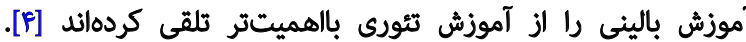

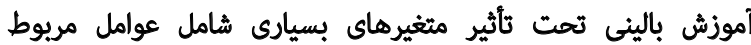

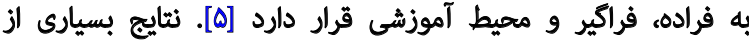

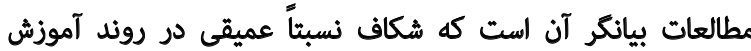

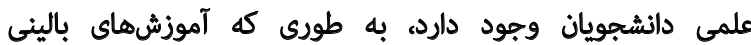

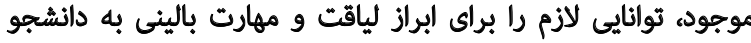

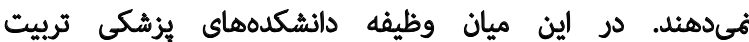

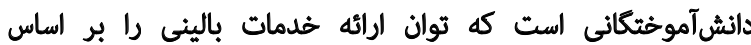

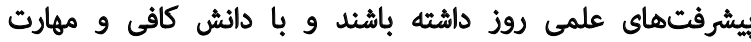
بالينى بتوانند نياز مددجويان رابر آورده كنيند.

مطالعات مختلف نشان دادهاند وجود مشكلات متعدد مانع دستيابى

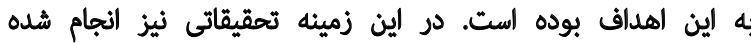

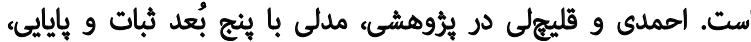

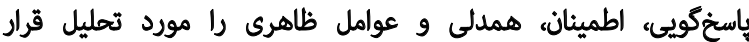

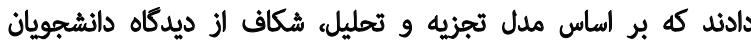

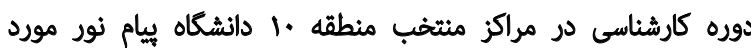

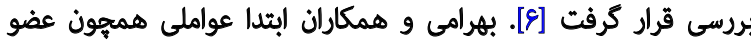

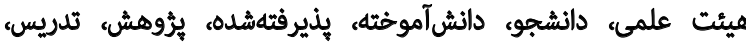

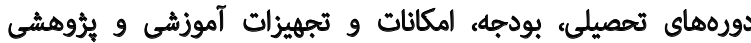

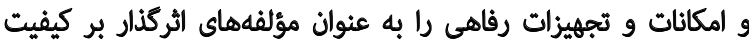

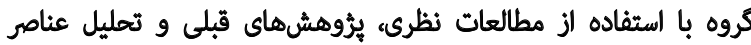

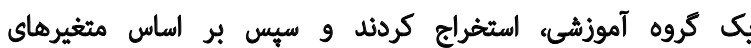
اثركذار در هر عامل و روابط بين آنها مدلى كلى آلى براى شبيهسازي

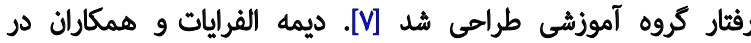

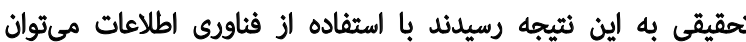

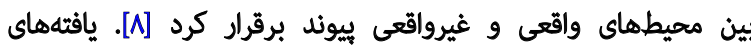

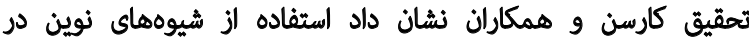

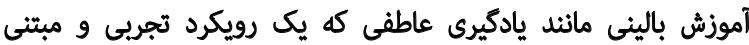

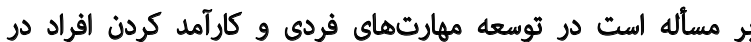

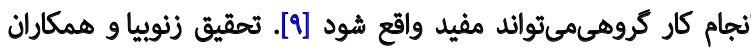
نشان داد يك برنامه مبتنى بر مفهوم و با الستفاده از الستراتثرىهاي

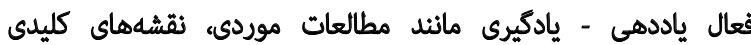

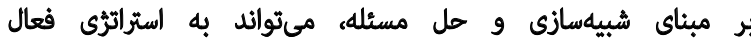

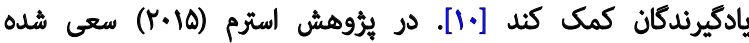

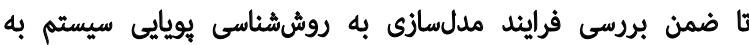

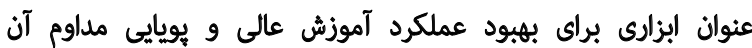
توجه شود و تغييراتى كه در انكيزهاى استادان و فراكيران ايجاد

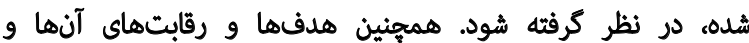

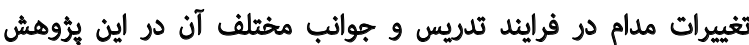

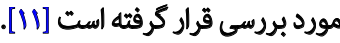


اين ئزوهش در محل بيمارستانهاى شهرستان ساوه انجام

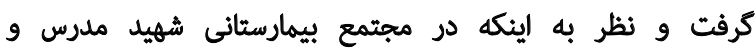

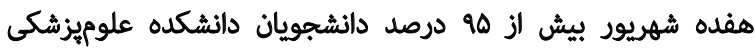

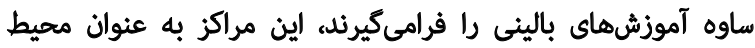

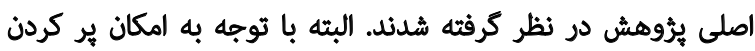

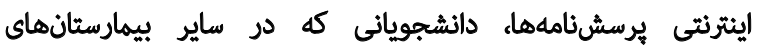

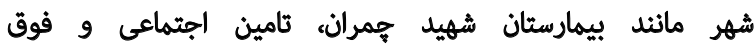

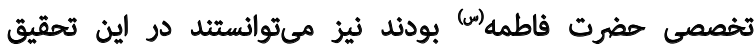
شركت كنند.

بعد الز النجام هر مصاحبه به هريك الز مصاحبهها كد داده

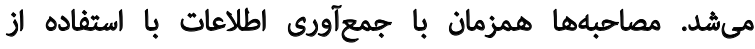

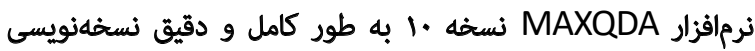

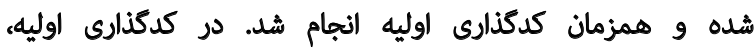

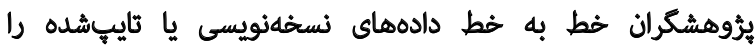

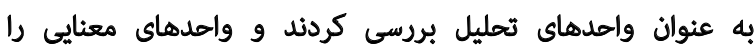

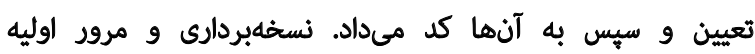

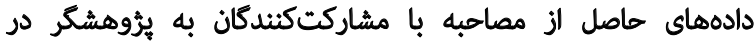

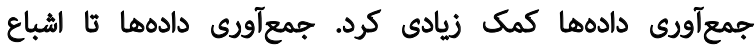
آن الدامه يافت، به طورى كه در مصاحبه با الساتيد و دانشجويان

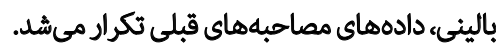

به منظور تعيين روايى صورى الز دو روش كيفى و كمى استفاده

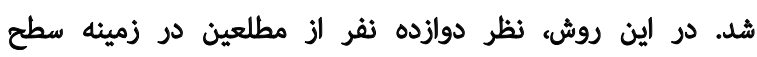

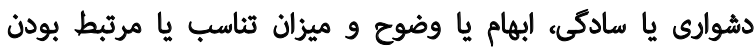

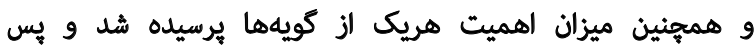

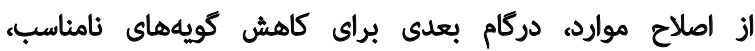
نظرات تيم تحقيق و افراد مطلع مورد استفاده قرار كرفت. جهت دارت

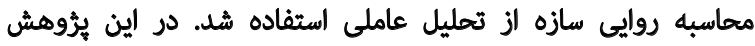
الز محاسبه ضريب همبستكى درونطبقهاى براى بررسى هائيايى

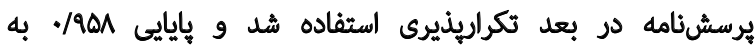
دست آمد كه عالى توصيف مي دشد.

براى تجزيه و تحليل دادههاى بيروهش از روش كراندد تئورى

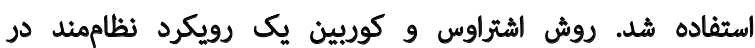

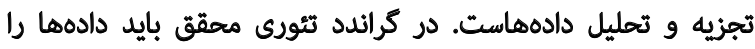

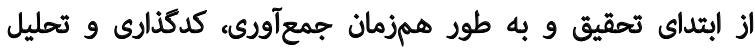

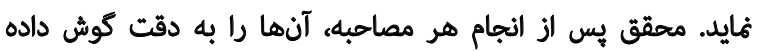

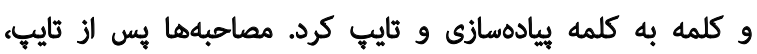

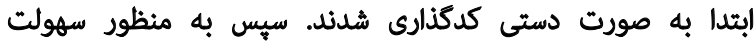

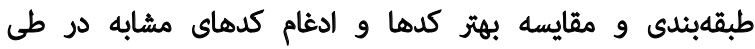

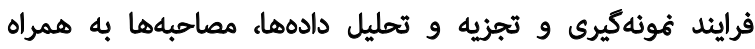

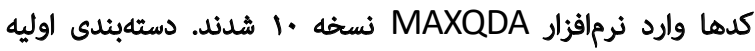

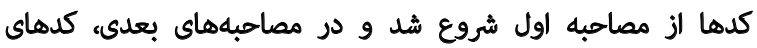

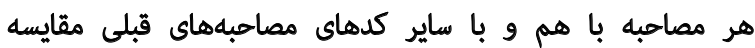

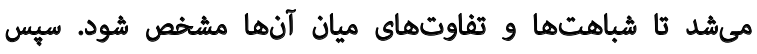

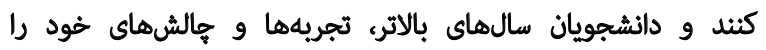

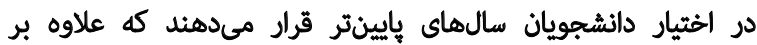

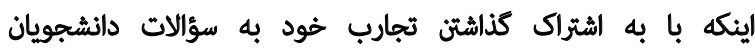

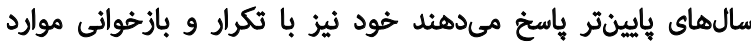

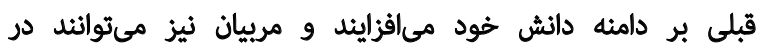

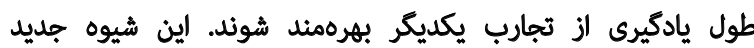

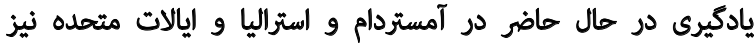

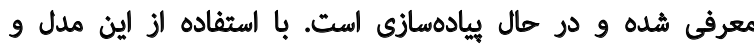
الكوى مشاركتي در يادكيرى طيف وسيعى از مربيان و دانشجويان

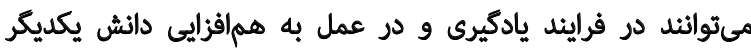
و تيم آموزشدهنده كمك فمايند [III] فئ.

با توجه به تحقيقات مختلفى كه هم در ايران و هم در ساير

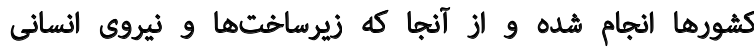

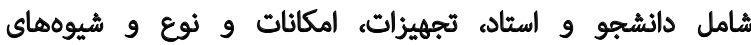

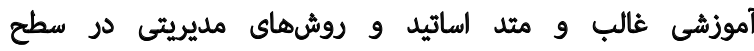

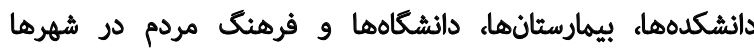

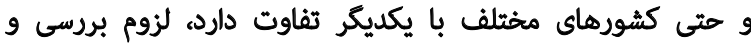

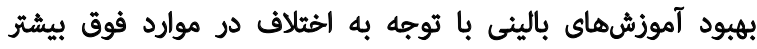

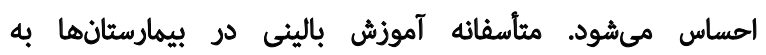

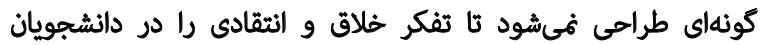

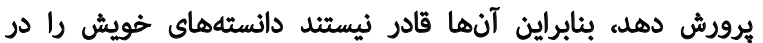

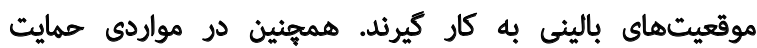

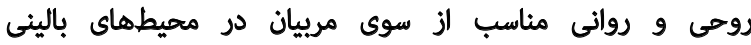

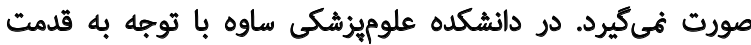

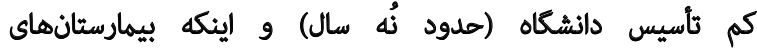

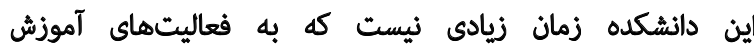
بالينى مشغول هستند، هنوز تحقيقى در مورد كيفيت آموزشيى زئي

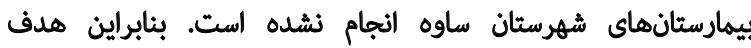

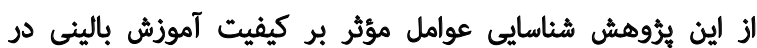

بيمارستانهاي شهر ساوه بود.

$$
\text { 10. }
$$

اين هُروهش براى شناخت بهيده، با دادههاى كيفى شروع شد و

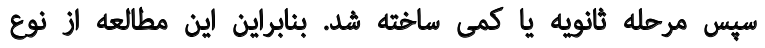
مطالعات تركيبى و در زمره طرحهاى اكتشافى الست. در مرحله كيفى دئى

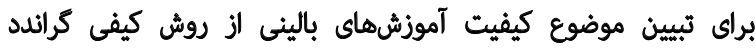

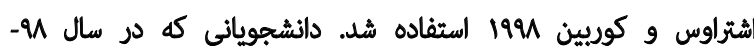

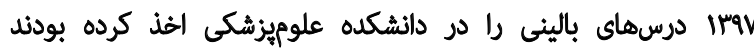

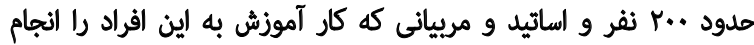

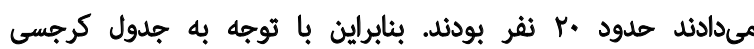

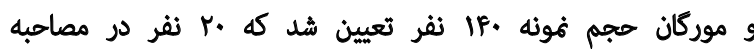

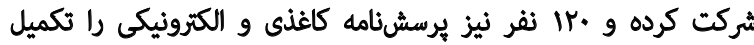

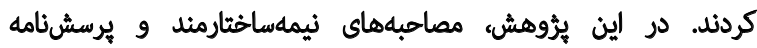

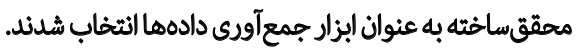


با توجه به مقدار واريانس تييينشده توسط عوامل معلوم شد

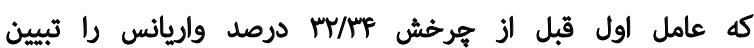

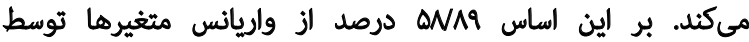

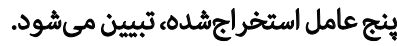

در اين بُوهش هون در هاختار و ابزار ئزوهش، تنها يك عامل

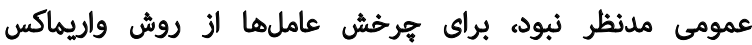
استفاده شد (جدول شماره مان). بعد الز جرخش، الززشهاى ويرّه مؤلفهها به كونه يكنواخت برى برى

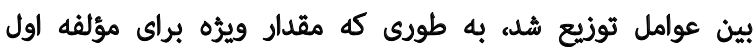

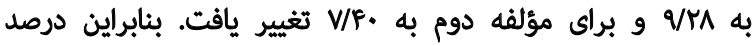

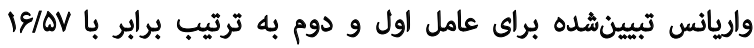

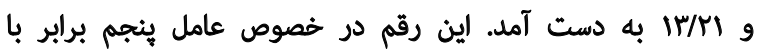
NT.

يزؤشكران به منظور تحقيق درباره ماهيت روابط بين مثغيرها

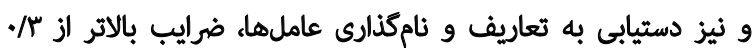

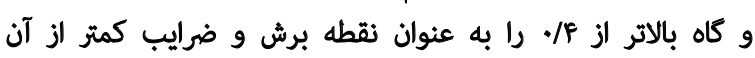

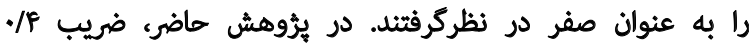

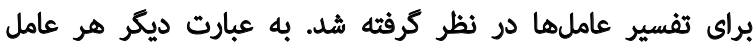

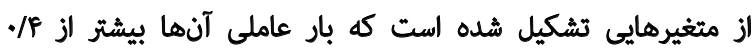

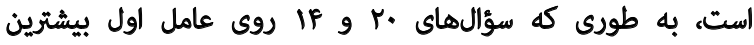
بار عاملى رابه خود اختصاص دالدند (جدول شماره ؟).

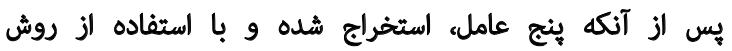

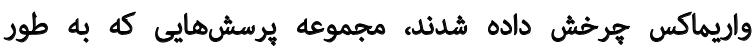
مشترك با يك عامل همبسته بودند، به شرح زير به دست آمدند:

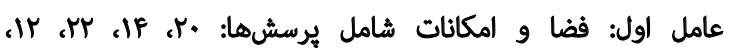

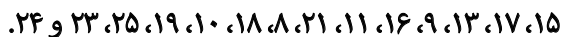

عامل دوم: بهبود فرايندهاى آموزشى شامل يرسشهاى

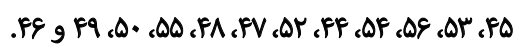

عامل سوم: كمبودهاى آموزشى و مديريتى شامل كويههاى

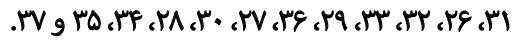
عامل جهارم: الرزيابى استاد، دربركيرنده سؤالهاى "ا، ه، ا، "، $. r g 8 . V$
كدها بر اساس شباهتهايى كه با يكديكر داشتند در يك طبقه

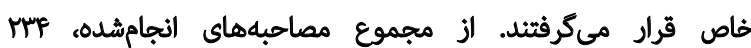

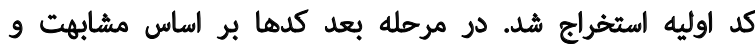

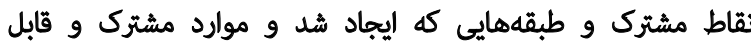

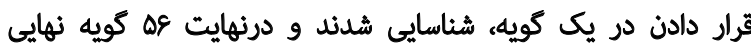
استخراج شمد. Ladị̂ำ

قبل الز اجراى تحليل عاملى و شناسايى عوامل لازم است دو

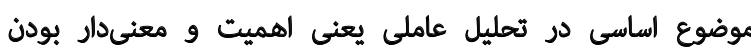

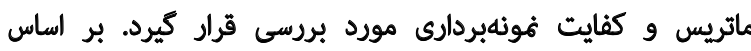

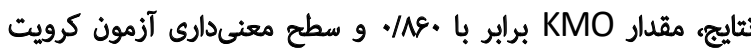

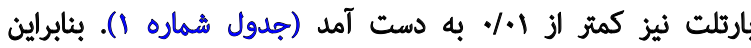

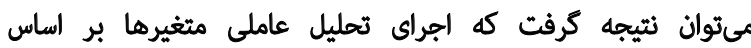
ماتيس همبستكى هامل در كروه مورد مطالعه ثابل توحيه أسيث. به منظور استخراج عوامل اوليه، از روش تحليل مولفههاى اصلى زيلى

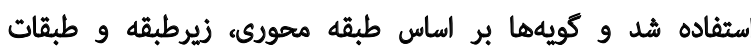
فرعى تقسيمبندى شدند (جدول شهماره r).

در ارزيابى اوليه، وه عامل در مدل منظور شد كه عامل اول با درو بال

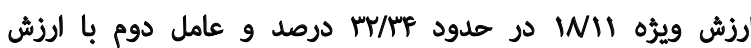

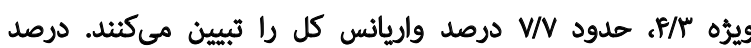

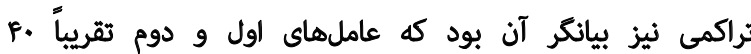
درصد واريانس متغيرها راتبيين مىثمايند (جدول شماره بان).

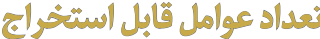

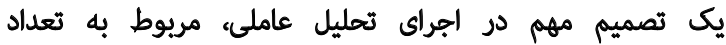

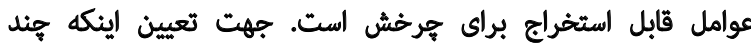

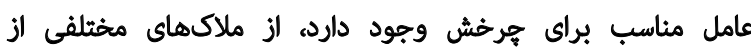

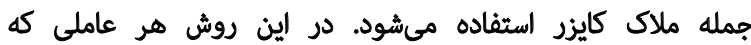

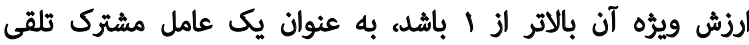

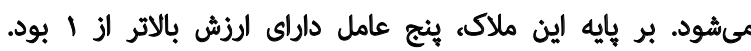

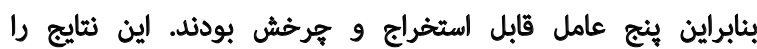

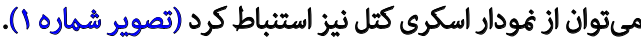
جدول ا. نتايج آزمون KMO و آزمون كرويت بارثلت

\begin{tabular}{|c|c|c|}
\hline .117. & & \\
\hline$p q . / 1$ & خیدو & \\
\hline laf. & درجه آزادى & آزمون كرويت بارثلت \\
\hline$<\% 1$ & سطح معنى الارى & \\
\hline
\end{tabular}


جدول r. تقسيمبندى كويهها بر اساس طبقه محورى، زيرطبقه و طبثات فرعى

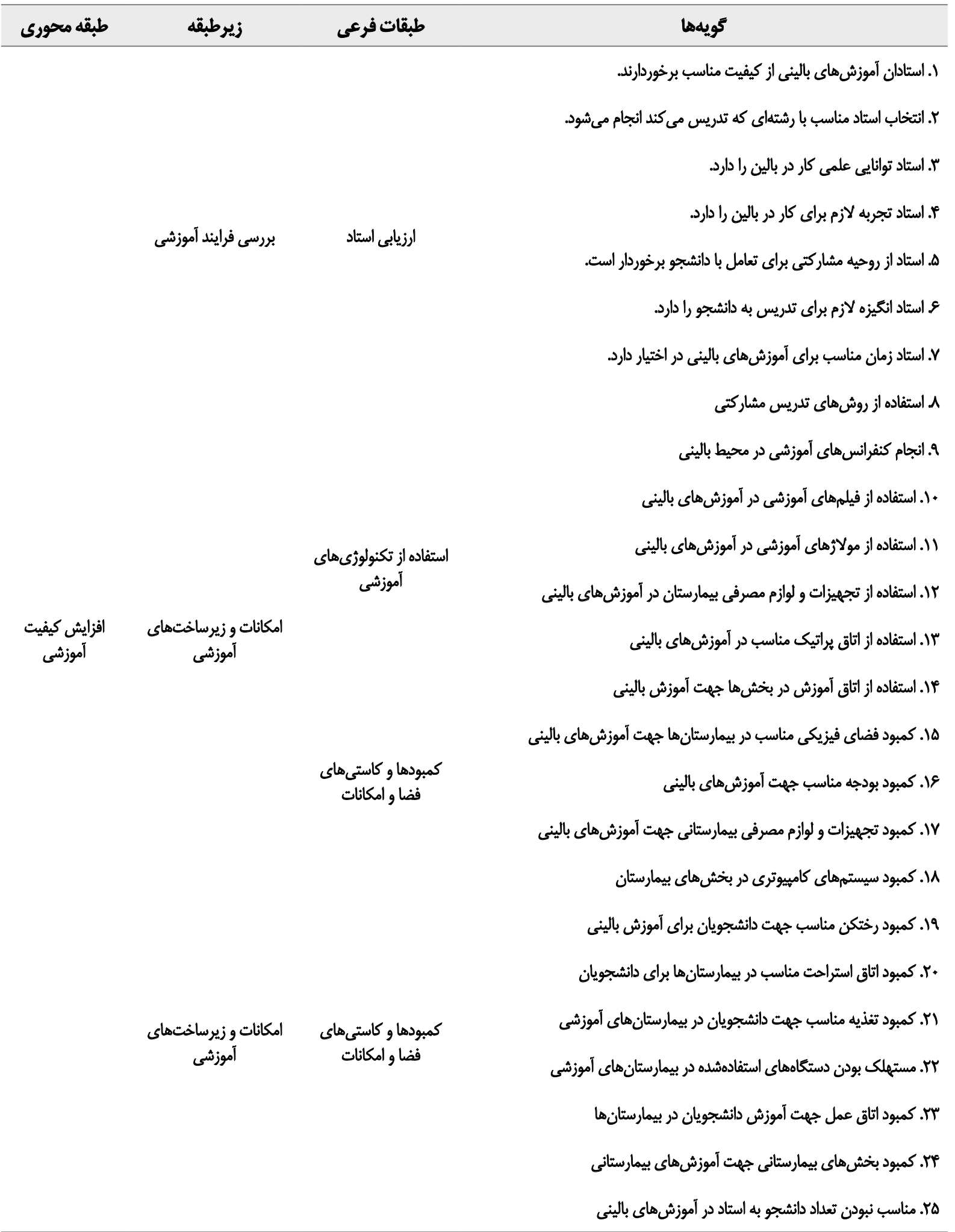




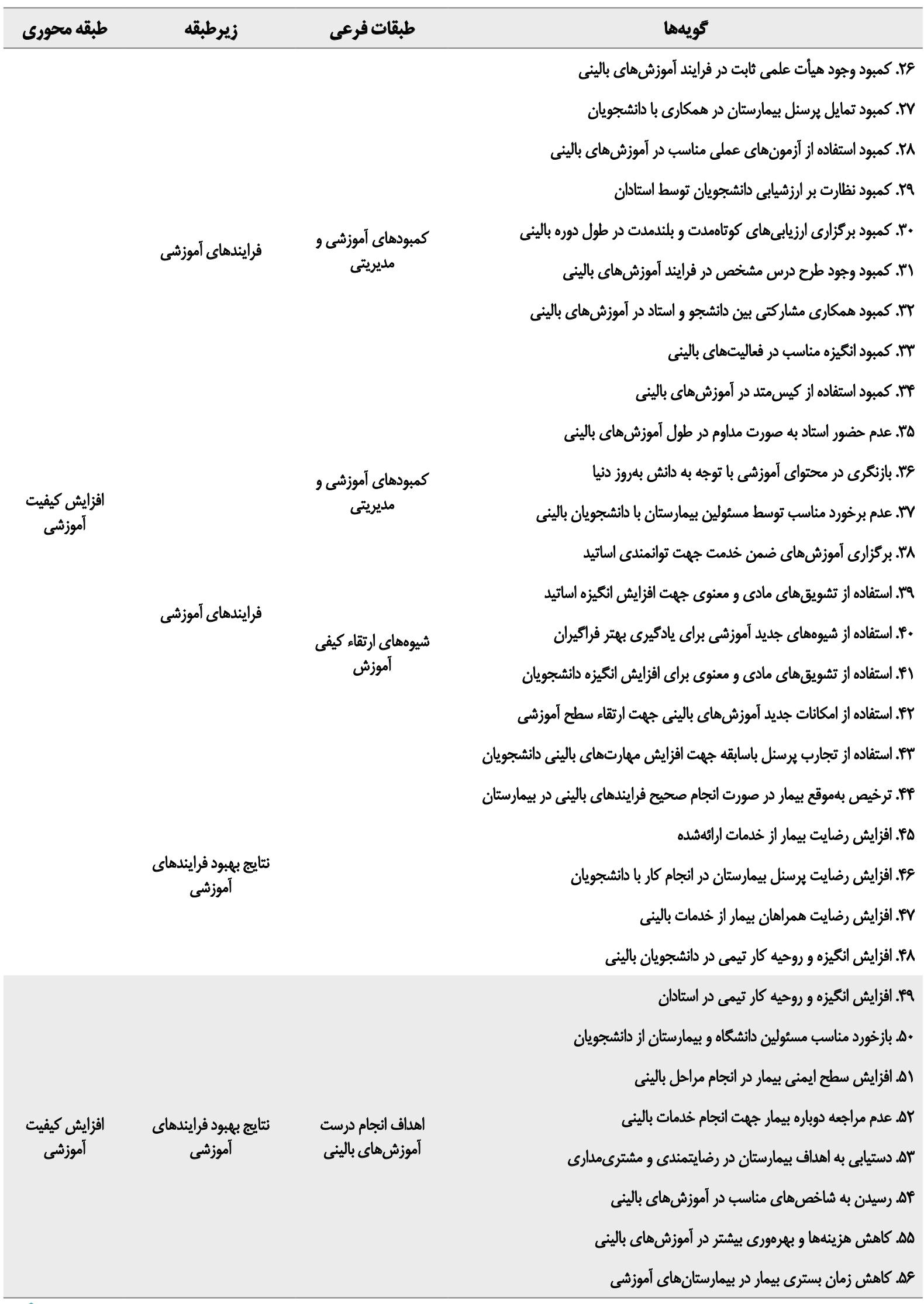


جدول r. مشخصهماى آمارى اوليه يرسشنامه به روش PC

\begin{tabular}{|c|c|c|c|}
\hline عاملها & ارزّ ويثُه & درود واريانس & درصد تراكمى \\
\hline$M T / M T$ & $r r / M P$ & WII & 1 \\
\hline$r+1+r$ & $V / \& A$ & $r / r$ & $r$ \\
\hline PVIEY & $V(8)$ & r/Ye & $r$ \\
\hline$\Delta r / A q$ & $g / r V$ & $r(\Delta)$ & r \\
\hline$\Delta N 19$ & $\Delta$ & $V / A$. & $\Delta$ \\
\hline SI/MT & T/R &.$/ 99$ & 8 \\
\hline erig. & $T / K A$ &.$/ 4$ & $\checkmark$ \\
\hline $8 \Delta / V$ & $r /$ &.$/ 9 \Delta$ & $\wedge$ \\
\hline EVIOA & $1 / M$ &.$/ 9 r$ & 9 \\
\hline EA/TE & $\mathrm{I} / \mathrm{MA}$ &.$/ 91$ & 1. \\
\hline$n / \circ f$ & $1 / 8 A$ &.$/ M A$ & 11 \\
\hline$r / e V$ & V/Fr & . INE & ir \\
\hline$V P / T \Delta$ & $1 / \Delta A$ & - /Ar & זו \\
\hline $\mathrm{V} \Delta / \mathrm{W}$ & V/ar & $\cdot|A|$ & if \\
\hline$W / r I$ & $1 / 4 F$ & $\cdot / A$ & 10 \\
\hline WNEI & $1 / 4$ & $\cdot / V A$ & 18 \\
\hline $\mathrm{V} / \mathrm{M}$ & $1 / \pi V$ & $\cdot / n$ & iv \\
\hline $11 / 11$ & זיו & .189 & $M$ \\
\hline AT/MT & $M / T$ &.$/ 81$ & 19 \\
\hline Ar/trV & $1 / 10$ & .180 & r. \\
\hline$A F / \Delta \Delta$ & $1 / .9$ & .181 & ri \\
\hline$A \Delta / \Delta P$ & 1 &.$/ \Delta F$ & Mr \\
\hline$N E / P V$ &.$/ 91$ & $\cdot|\Delta|$ & $m$ \\
\hline$A V / r r$ & $\cdot \mathbb{N}^{2}$ & . / FA & $M$ \\
\hline WIS & . &.$/ F$ & ro \\
\hline WMA & -/Ar &.$/ 48$ & re \\
\hline $19 / \mathrm{W}$ & $+/ 199$ & / $/ F F$ & Tr \\
\hline $9+/ 4 a$ & $\cdot M$ &.$/ 4$ & rA \\
\hline $91 / 18$ & +189 &.$/ r V$ & rq \\
\hline $91 / \mathrm{W}$ & . & $\cdot / r \Delta$ & r. \\
\hline $97 / \% q$ & - IAY &.$/ M$ & II \\
\hline $9 \% / 9 V$ & $.1 \Delta 1$ &.$/ M T$ & MT \\
\hline
\end{tabular}




\begin{tabular}{|c|c|c|c|}
\hline عاملها & ارزش ويثره & درصد واريانس & درصد تراكمى \\
\hline QY/QY & $\cdot / \Delta \Delta$ &.$/ M$ & $\pi$ \\
\hline $9 \% / .8$ &.$/ \Delta P$ &.$/ r$ & $\pi$ \\
\hline $9 \varphi / \Delta Y$ &.$|\Delta|$ & ./ra & ro \\
\hline $9 \$ / .8$ &.$/ F A$ &.$/ T V$ & ( ) \\
\hline $9 ه / F A$ & $* / \pi$ & $\cdot / M F$ & r \\
\hline $9 \Delta / M$ & $\cdot / 4$ &.$/ 4 r$ & ra \\
\hline$\vartheta / T V$ & $+/ / 4 q$ & $\cdot / M$ & rq \\
\hline QIET &.$/ 28$ &.$/ 4$ & f. \\
\hline$\vartheta / Q$ &.$/ 20$ & .119 & il \\
\hline $9 \vee /{ }^{\circ}$ &.$/ m$ & .111 & RT \\
\hline qY/EY & $\cdot / / 1$ &.$/ 1 \mathrm{~V}$ & Pr \\
\hline จพ/9. & - /ra & .118 & Pf \\
\hline WIS & ./Y & .110 & PQ \\
\hline WTF & / &.$/ 1 F$ & is \\
\hline QWef & A $/ M$ &.$/ 14$ & pr \\
\hline WNe & . &.$/ 1 r$ & ra \\
\hline $99 / 1$ & H/Tr &.$/ 1 T$ & pq \\
\hline $99 / 7 \%$ &.$/ 19$ & .11 & $\Delta$. \\
\hline $99 / 45$ &.$/ 18$ & .1 .9 & $\Delta 1$ \\
\hline १ด/ฝ &.$/ 1 f$ & $.1 \cdot 1$ & ar \\
\hline $99 / 89$ & ./Ir & $.1+V$ & $\Delta r$ \\
\hline १ด/ภ1 &.$/ 1 r$ & $+1 \cdot v$ & af \\
\hline १จ/१ & +11 & .1 .8 & $\Delta \Delta$ \\
\hline $1 .$. & .1 .9 & $.1 . \Delta$ & $\Delta$ \\
\hline
\end{tabular}

از تجهيزات و لوازم مصرفى بيمارستانها با نمره وبش/• بيشترين

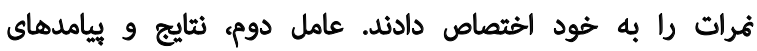

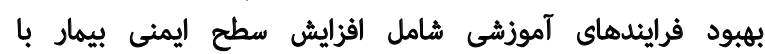

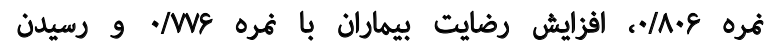

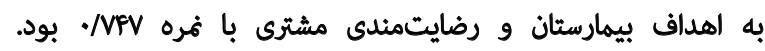

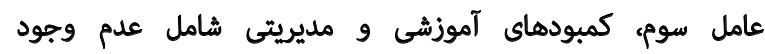

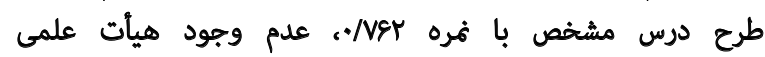

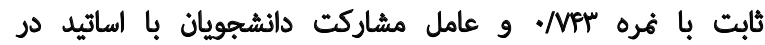

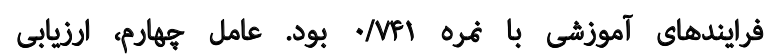

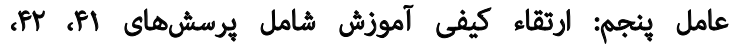

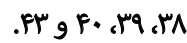
\&es

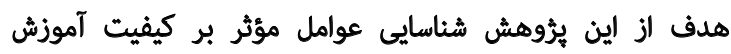

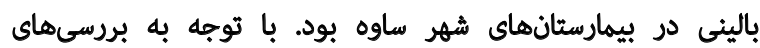

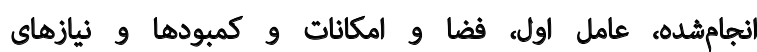

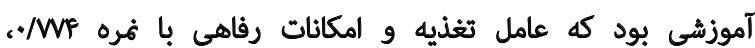

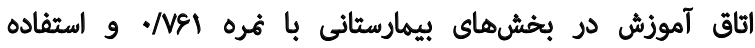




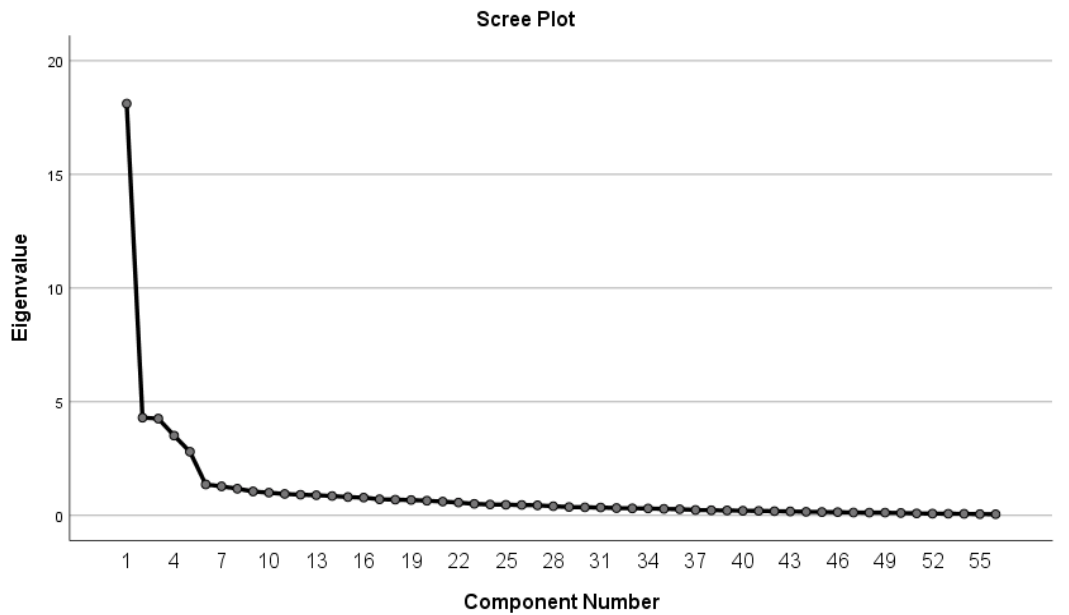

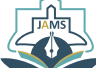

مشخص شد كه انجام درست فرايندهاى بالينى مىثواند به ايمنى مانى

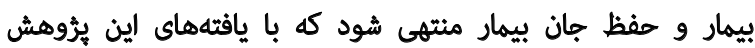

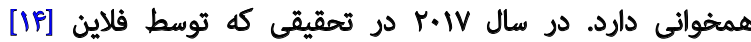

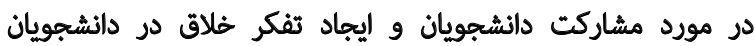

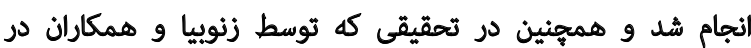

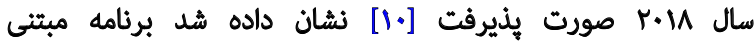

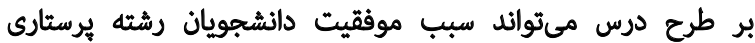

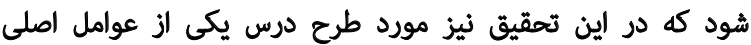

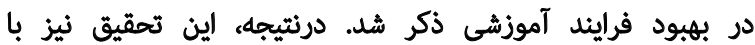

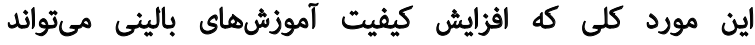

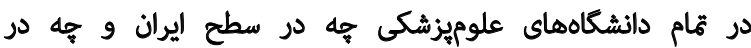

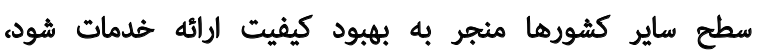
همخوانى دارد، وألى هون المكانات، تجهيزات، نيروها و ونهات ساختار و حتى فرهنكهاي غالب در هر دانشكاه با يكديكر تفاوت دارئ دارد

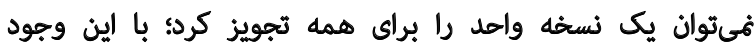

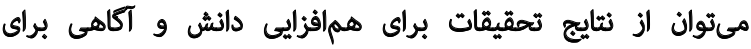
ساير تحقيقات الستفاده كرد.
تصوير ا. نمودار شيبدار حاصل از تحليل عاملى ثرسشنامه عهسؤلى

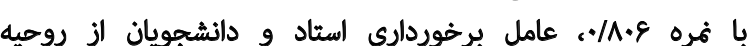

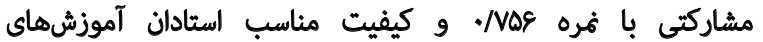

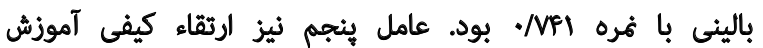

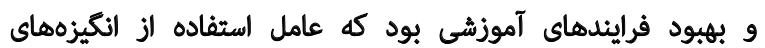

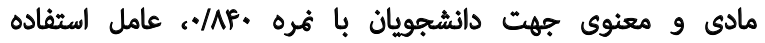
از امكانات جديد آموزشى درى آموزشهاى بالينى با غهره

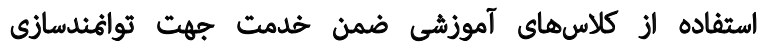

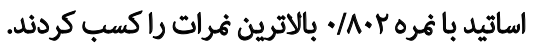

اين تحقيق با تحقيقاتى كه در مورد ابعاد مختلف بهبود كيفيت

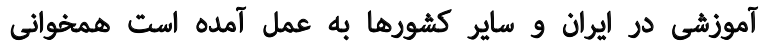

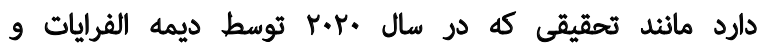

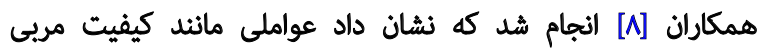

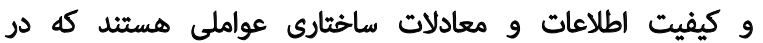

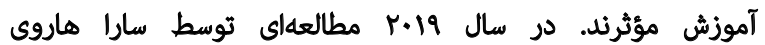

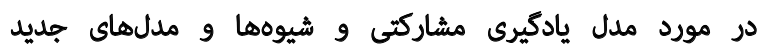

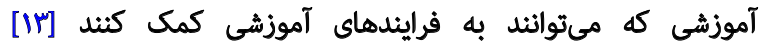

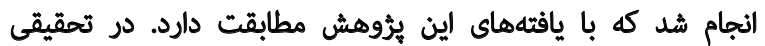

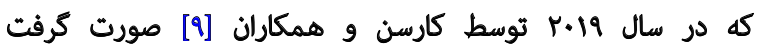

جدول P. ماتريس عاملي جرخشنايافته مجموعه عه سؤالي با روش pC

\begin{tabular}{|c|c|c|c|}
\hline درصد تراكمي & مرود واريانس & الرزش ويزوه & عاملها \\
\hline$M T / M$ & $M / M P$ & WNI & 1 \\
\hline$p \cdot l \cdot r$ & V/FA & $f / r$ & $r$ \\
\hline PWET & Vle. & r/TE & $r$ \\
\hline$\Delta r / A 9$ & e/rV & $r / \Delta \mid$ & p \\
\hline$\triangle N A 9$ & $\Delta$ & $1 / A$. & $\Delta$ \\
\hline
\end{tabular}


جدول هـ مشخصهاى آمارى براى بينج عامل استخراجشده بعد از جرخش واريماكس

\begin{tabular}{|c|c|c|c|}
\hline درصد تراكمى & درصد واريانس & ارزش ويرٔه & عاملها \\
\hline $\mid g / \Delta V$ & $\mid g / \Delta V$ & q/TA & اول \\
\hline$r q / r \wedge$ & $|r / T|$ & $V / f$. & دوم \\
\hline$P r / \cdot V$ & $I T / T q$ & S/M & سوم \\
\hline 0.189 & NAT & $r / A r$ & جهارم \\
\hline QNAq & NT. & $P / \Delta q$ & ئجمم \\
\hline
\end{tabular}

5

جدول \&. ماتريس عاملى، عامل هاى جرخش يافته مجموعه \&هؤالى با شيوه واريماكس

\begin{tabular}{|c|c|c|c|c|c|}
\hline عامل ينجم & عامل جهارم & عامل سوم & عامل دوم & عامل اول & سؤالات \\
\hline & & & &.$/ M^{e}$ & r. \\
\hline & & & &.$N 81$ & If \\
\hline & & & & Mrq & M \\
\hline & & & & - MIV & ir \\
\hline & & & & $.18 \lambda$ & 10 \\
\hline & & & & - IEVA & iv \\
\hline & & & & $.18 v^{6}$ & r \\
\hline & & & & - lear & 9 \\
\hline & & & & $.18+8$ & 18 \\
\hline & & & & .194 & 11 \\
\hline & & & & . & M \\
\hline & & & & . Iatr & 1 \\
\hline & & & &.$|8|$ & M \\
\hline & & & & $.18 \cdot r$ & 1. \\
\hline & & & & $.18 .$. & 19 \\
\hline & & & & . IAAF & rA \\
\hline & & & &.$/ A A F$ & r \\
\hline & & & & low & if \\
\hline & & & $\cdot 1 A \cdot 8$ & & $\Delta 1$ \\
\hline & & & . MV & & $P Q$ \\
\hline & & & - MFV & & $\Delta H^{\omega}$ \\
\hline & & &.$M T F$ & & $\Delta F$ \\
\hline & & & 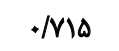 & & of \\
\hline & & &. $\mid V \cdot 1$ & & pif \\
\hline
\end{tabular}




\begin{tabular}{|c|c|c|c|c|c|}
\hline عامل بنجمم & عامل جههارم & عامل سوم & عامل دوم & عامل اول & سورالات \\
\hline & & & . IEAT & & $\Delta r$ \\
\hline & & & - $|8 A|$ & & PV \\
\hline & & & $.189 V$ & & Pa \\
\hline & & & .1880 & & $\Delta \Delta$ \\
\hline & & & IEPV & & Q. \\
\hline & & &.$/ 1999$ & & $p q$ \\
\hline & & & .larr & & pe \\
\hline & & - Ner & & & m \\
\hline & & . MrT & & & re \\
\hline & & $+M+1$ & & & rT \\
\hline & & $\cdot M T$ & & & $m$ \\
\hline & & $\cdot N \cdot V$ & & & rq \\
\hline & & $.18 \mathrm{Va}$ & & & rg \\
\hline & & - legr & & & $r$ \\
\hline & & $.18 \Delta$ & & & r. \\
\hline & & . Igra & & & ra \\
\hline & & $.18 T V$ & & & $\pi$ \\
\hline & & . & & & هם \\
\hline & &.$/ 499$ & & & rv \\
\hline & $\cdot 11 \cdot 8$ & & & & $p$ \\
\hline & - IVAS & & & & $\Delta$ \\
\hline & $\cdot / N q$ & & & & 1 \\
\hline & . Mre & & & & $r$ \\
\hline & +MTr & & & & $\checkmark$ \\
\hline & $+/ M T r$ & & & & 8 \\
\hline & .184 & & & & $r$ \\
\hline ./AF. & & & & & il \\
\hline - /ATA & & & & & pr \\
\hline$\cdot \mid A \cdot r$ & & & & & ra \\
\hline.$/ 199$ & & & & & mq \\
\hline.$M$ & & & & & f. \\
\hline$+/ V+1$ & & & & & $r$ \\
\hline
\end{tabular}


ملاحظات اخلاقي

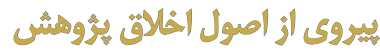

اين مقاله طبق نامه شهاره

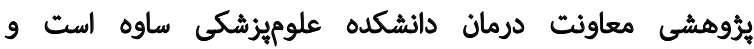

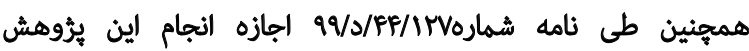
در دانشكده علوميزشكى ساوه داده شده است.

$$
\text { Ito nols }
$$

اين مقاله الز رساله دكترى نويسنده الوله در كروه مديريت

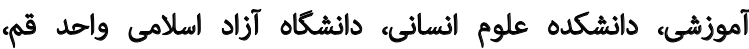

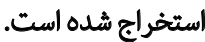

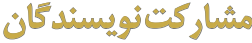

مفهومسازى: سيدمصطفى مرتضوى، غلامرضا شريفى رادي؛ روش

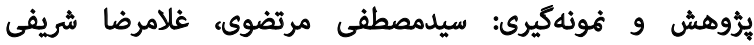

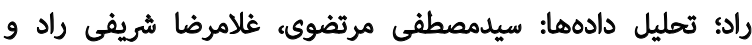
سيامك محبى: نكارش متن و بازبينى: تمام نويسندكان.

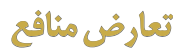
نويسندكان مقاله هيجكونه تعارضى در منافع اعلام نكردند.

\section{تتيجليرى}

اولين عامل، عامل فضا و امكانات است و رفع كمبودهاى

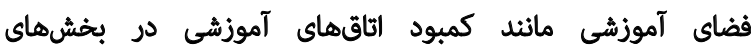

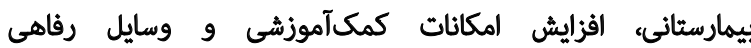

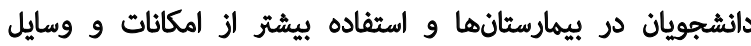

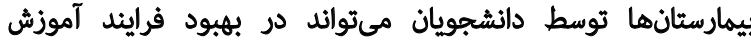
و بهبود كيفيت ارائه خلمات به مردم مفيل باشل.

دومين عامل و يكى الز مهمترين عوامل در افزايش كيفيت

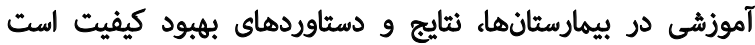

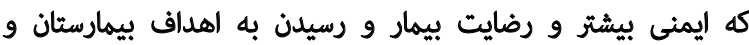
مشترىمدارى بيشترين نمرات راكسب كردند.

سومين عامل، كمبودهاى آموزشى و مديريتى است به به طورى

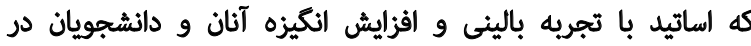

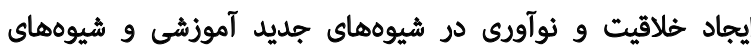

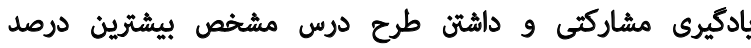

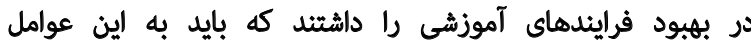

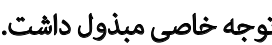

جهارمين عامل مؤثر، عامل الرزيابى صصيح اساتيد از دانشجويان

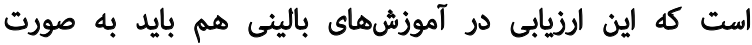

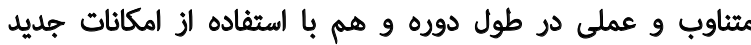

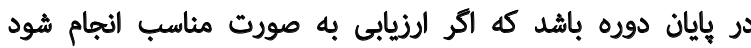
مى مواند در بهبود فرايندهاى آموزشى تاثيركذار باشد.

ينجمين عامل نيز الرتقاى كيفى آموزش شامل إيجاد انكيزه

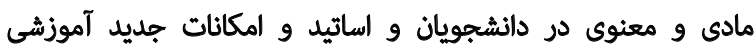

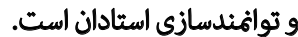

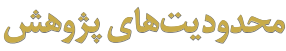
الف) محدوديتهايى كه در اختيار يزووهشكر بودند:

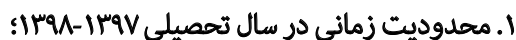
r. محدوديت مكانى كه ئورهش محدود به بيمارستانهاى دانشكده علوميزشكى ساوه بوده است. ب) محدوديتهايى كه در اختيار يُروهشكر نبودند: ا. دسترسى مهلود به افراد متخصص و مجرب دري حوزئ

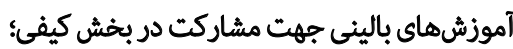
r. تفاوتهاى علمى و تجربههاى مختلفى كه مشاركتكنندكان مريخوهش داشتند؛

". وجود تفاوتهاى فردى (از جمله ميزان علاقه به تحقيق). 


\section{References}

[1] Creswell JW, Plano Clark VL. Designing and conducting mixed methods research. $2^{\text {th }}$ ed. Thousand Oaks, CA: Sage; 2011. https://books. google.com/books/about/Designing_and_Conducting_Mixed_ Methods_R.html?id=6tYNoOUpEqkC

[2] Mayoh J, Onwuegbuzie AJ. Toward a conceptualization of mixed methods phenomenological research. J Mix Methods Res. 2015; 9(1):91-107. [DOI:10.1177/1558689813505358]

[3] Creswell JW, Klassen AC, Plano Clark VL , Smith KC. Best practices for mixed methods research in the health sciences. The Nature and Design of Mixed Methods Research. 2011:1-37. https://www.csun.edu/sites/ default/files/best_prac_mixed_methods.pdf

[4] Lindlof TR, Taylor BC. Qualitative Communication Research Methods. $2^{\text {th }}$ ed. Thousand Oaks: Sage; 2002. https://openlibrary.org/ books/OL3553314M/Qualitative_communication_research_ methods.\#edition-details

[5] Streubert J, Carpenter DR. Qualitative research in nursing: Advancing the Humanistic Imperative. $3^{\text {th }}$ ed. Philadelphia: Lippincott Williams \& Wilkins; 2003. https://books.google.com/books/about/Qualitative_ Research_in_Nursing.html?id=8poAQgAACAAJ

[6] Ahmadi SAA, Ghelichi B. [Designing educational services of quality management model in Payame Noor University (Persian)]. Payame Noor Journal. 2003; 3(3):79-100. https://www.sid.ir/fa/journal/ViewPaper. aspx?id=127443

[7] Bahrami A, Yadegarzadeh G, Paran K. [Policy-making and factors affecting internal evaluation of departments: A system dynamics approach (Persian)]. IRPHE. 2007; 13(2):49-77. http://journal.irphe.ac.ir/article1-79-en.html

[8] Al-Fraihat D, Joy M, Sinclair J. Evaluating E-learning systems success: An empirical study. Comput Human Behav. 2020; 102:67-86. [DOI:10.1016/j.chb.2019.08.004]

[9] Carson OM, Laird EA, Reid BB, Deeny PG, McGarvey HE. Enhancing teamwork using a creativity-focussed learning intervention for undergraduate nursing students-a pilot study. Nurse Educ Pract. 2018; 30:20-6. [DOI:10.1016/j.nepr.2018.02.008] [PMID]

[10] Ma PHX, Chan ZCY, Loke AY. Conflicting identities between sex workers and motherhood: A systematic review. Women Health. 2019; 59(5):53457. [DOI:10.1080/03630242.2018.1500417] [PMID]

[11] Stern E. Editorial. Evaluation. 2015; 21(2):131-4. [Doi: $10.1177 / 1356389015580670]$

[12] Berliner DC. MCLB (Much Curriculum Left Behind): A US calamity in the making. Educ Forum. 2009; 73(4):284-96. [DOI. org/10.1080/00131720903166788]

[13] Berliner D. Rational responses to high stakes testing: The case of curriculum narrowing and the harm that follows. Cambridge J Educ. 2011; 41(3):287-302. [DOI:10.1080/0305764X.2011.607151]

[14] Harvey S, Uren CD. Collaborative learning: Application of the mentorship model for adult nursing students in the acute placement setting. Nurse Educ Today. 2019; 74:38-40. [DOI:10.1016/j.nedt.2018.11.022] [PMID] 